\title{
Learning Causes Reorganization of Neuronal Firing Patterns to Represent Related Experiences within a Hippocampal Schema
}

\author{
Sam McKenzie, Nick T. M. Robinson, Lauren Herrera, Jordana C. Churchill, and Howard Eichenbaum \\ Center for Memory and Brain, Boston University, Boston, Massachusetts 02215
}

According to schema theory as proposed by Piaget and Bartlett, learning involves the assimilation of new memories into networks of preexisting knowledge, as well as alteration of the original networks to accommodate the new information. Recent evidence has shown that rats form a schema of goal locations and that the hippocampus plays an essential role in adding new memories to the spatial schema. Here we examined the nature of hippocampal contributions to schema updating by monitoring firing patterns of multiple CA1 neurons as rats learned new goal locations in an environment in which there already were multiple goals. Before new learning, many neurons that fired on arrival at one goal location also fired at other goals, whereas ensemble activity patterns also distinguished different goal events, thus constituting a neural representation that linked distinct goals within a spatial schema. During new learning, some neurons began to fire as animals approached the new goals. These were primarily the same neurons that fired at original goals, the activity patterns at new goals were similar to those associated with the original goals, and new learning also produced changes in the preexisting goal-related firing patterns. After learning, activity patterns associated with the new and original goals gradually diverged, such that initial generalization was followed by a prolonged period in which new memories became distinguished within the ensemble representation. These findings support the view that consolidation involves assimilation of new memories into preexisting neural networks that accommodate relationships among new and existing memories.

\section{Introduction}

Recent studies on memory consolidation have explored the mechanisms by which the hippocampus supports the assimilation of new experiences into preexisting networks of knowledge, called schemas (for review, see McKenzie and Eichenbaum, 2011). The important role of schemas in memory formation was introduced to cognitive science in the classic studies of Piaget (1926) and Bartlett (1932), who proposed that new memories that are consistent with preexisting knowledge are readily assimilated within existing memory networks, often requiring updating or modification of the existing schema to accommodate the new information. McClelland et al. (1995) introduced the idea that the hippocampus supports schema assimilation and accommodation in an influential model of hippocampal-cortical interactions during consolidation. Subsequent experimental evidence indicates that hippocampal-dependent consolidation is speeded when new memories are incorporated within a schema (Tse et al., 2007; van Kesteren et al., 2012), that hippocampal-dependent schemas support inferential memory expression (Bunsey and

Received Feb. 27, 2013; revised May 10, 2013; accepted May 12, 2013.

Author contributions: S.M. and H.E. designed research; S.M., N.T.M.R., L.H., and J.C.C. performed research; S.M. analyzed data; S.M. and H.E. wrote the paper.

This work was supported by National Institute of Mental Health Grant MH52090.

The authors declare no competing financial interests.

Correspondence should be addressed to Howard Eichenbaum, 2 Cummington Street, Center for Memory and Brain, Boston University, Boston, MA 02215. E-mail: hbe@bu.edu.

DOI:10.1523/JNEUROSCI.0879-13.2013

Copyright $\odot 2013$ the authors $\quad 0270-6474 / 13 / 3310243-14 \$ 15.00 / 0$
Eichenbaum, 1996; Dusek and Eichenbaum, 1997; Heckers et al., 2004; Zeithamova and Preston, 2010), and that prefrontal-hippocampal interactions are critical to schema formation, modification, and expression (Tse et al., 2011; Zeithamova et al., 2012). Furthermore, the phenomenon of reconsolidation may reflect the updating of a preexisting schema (Hardt et al., 2010; McKenzie and Eichenbaum, 2011; Dudai, 2012).

Despite a wealth of evidence that the hippocampus plays a central role in schemas, the mechanism by which neuronal networks in the hippocampus integrate memories into schemas has received little attention. Indirect evidence has come from studies that observe a "partial remapping" of hippocampal spatial firing patterns after alterations in salient spatial cues, which could reflect both assimilation of new information and accommodation of existing network representations (for review, see Eichenbaum, 1999). Several other studies have reported similarities in the firing patterns of hippocampal neurons associated with traversing similar routes through different mazes (Singer et al., 2010), with objects in different locations (Wood et al., 1999) or in different examples of the stimuli with the same meaning (Hampson et al., 2004; Quiroga et al., 2005). The common coding of events that are shared among memories could act as "nodes" that connect different memories within schematic networks. However, no studies have examined how common representations develop as new memories are added to a preexisting framework. In the present study, we extend previous findings on hippocampal neurons that develop firing patterns associated with goal locations when goals are moved in water mazes (Hollup et al., 2001) and open 
fields (Dupret et al., 2010), by exploring how the hippocampus represents the addition of new goals within an environment for which the spatial representation of preexisting goals has been characterized. Pursuing the neural representations that underlie characteristics of schemas described by Piaget (1929), we explored how new goals are assimilated into a preexisting hippocampal schema and how the original schema is modified to accommodate the new related information. The results show that a preexisting schema assimilates new goal memories while distinguishing preexisting memories and accommodates its structure to integrate the new knowledge within the preexisting network representation.

\section{Materials and Methods}

\section{Subjects}

Five male Long-Evans rats (Charles River Laboratories) were housed within the Laboratory Animal Care Facility on the Boston University Charles River Campus. Subjects were individually housed and kept on a $12 \mathrm{~h} \mathrm{light/dark} \mathrm{cycle.} \mathrm{During} \mathrm{behavioral} \mathrm{testing,} \mathrm{subjects} \mathrm{were} \mathrm{food} \mathrm{and}$ water deprived, ensuring a minimum of $85 \%$ free-feeding weight (300$500 \mathrm{~g}$ ) with access to $15 \mathrm{~min}$ of water per day. All animal protocols were approved by the Boston University Charles River Campus Institutional Animal Care and Use Committee.

\section{Surgery}

Rats were anesthetized using a mixture of $3 \%$ isoflurane in oxygen and were injected with atropine $(0.03 \mathrm{mg} / \mathrm{kg}$, s.c. $)$ and buprenorphine $(0.1$ $\mathrm{mg} / \mathrm{kg}$, s.c.) before surgery and meloxicam ( $1 \mathrm{mg} / \mathrm{kg}$, s.c. $)$ immediately after surgery. An ophthalmic ointment was applied to the eyes, and temperature was maintained between $37^{\circ} \mathrm{C}$ and $38^{\circ} \mathrm{C}$. Ringer's solution was injected to maintain hydration. Stainless steel bone screws, including two ground screws, were inserted into the skull. A 2.0-mm-diameter hole was drilled into the skull using a dental drill, and the dura was removed. The base of the microdrive was aimed at the CA1 region of the left dorsal hippocampus ( $-3.5 \mathrm{~mm}$ anteroposterior, $+1.9 \mathrm{~mm}$ mediolateral). The craniotomy was sealed with Kwik Sil (World Precision Instruments), the microdrive and ground wires were secured in place using dental acrylic (Henry Schein) and CandB Metabond (Parkell), and the surgical site was sutured shut.

\section{Electrophysiological recordings}

Individually movable 24 -tetrode microdrives were built in-house. Tetrodes were spun from $12.5 \mu \mathrm{m}$ nickel-chromium Kanthal fine wire (Sandvik) and gold plated for an impedance of $200 \mathrm{k} \Omega$ at $1 \mathrm{kHz}$. At the end of surgery, each tetrode was lowered $\sim 850 \mu \mathrm{m}$ into the brain. After $5 \mathrm{~d}$ of recovery, the tetrodes were lowered over 7-14 d toward the CA1 layer. After the experiments, $25 \mu \mathrm{A}$ of current was passed through each tetrode for $30 \mathrm{~s}$ before perfusion and histological confirmation of tetrode placement.

As reported previously (MacDonald et al., 2011), the electrical signal was referenced to a common skull screw and differentially filtered for single-unit activity ( $154 \mathrm{~Hz}$ to $8.8 \mathrm{kHz}$ ) and local field potentials (LFPs) $(1.5-400 \mathrm{~Hz})$. The amplified spikes from each wire were digitized at 40 $\mathrm{kHz}$, whereas the field potential was digitized at $1 \mathrm{kHz}$ and monitored with the Multineuron Acquisition Processor (Plexon). Individual pyramidal neurons were isolated using Offline Sorter (Plexon) by visualizing combinations of waveform features (peak valley, valley, peak, principal components, and timestamps) extracted from wires making up a single tetrode (i.e., "manual cluster cutting"). Single-neuron selectivity was verified by the interspike interval histograms that contained no successive spikes within a $2 \mathrm{~ms}$ refractory period. Single-neuron stability was verified by comparing cluster stability across a recording session.

\section{Behavioral paradigm}

Before surgery, water-deprived rats were trained to retrieve water at several locations on a symmetrical wooden circular track $(107 \mathrm{~cm}$ in diameter) that contained 20 potential reward sites. The reward sites were small water dishes with adjacent LEDs connected by an angle bracket. The location of the goal for each trial was chosen pseudorandomly among four to five evenly spaced locations, with the goal never repeating more than three consecutive times. Each trial began with a blinking LED (300 $\mathrm{ms}$ on, $700 \mathrm{~ms}$ off) at the selected location, and, when the rat was within $15 \mathrm{~cm}$ of an LED-cued goal location, the light was held on for $5 \mathrm{~s}$, after which $75 \mu \mathrm{l}$ of water was delivered from the adjacent water port. If the rat did not wait at the reward site within $2 \mathrm{~min}$, there was a $15 \mathrm{~s}$ intertrial interval, and then the task progressed to the next trial.

After surgery, rats were retrained on the LED-cued version of the task. When tetrodes were positioned in dorsal CA1, three new spatially defined goal locations (SP1, SP2, SP3) were introduced over the course of 1 week; these goal locations were not cued by an LED and could only be identified by memory of their location relative to extra-maze cues (overhead lights and large white cue card). On day 1, 30 LED-cued trials were presented, and then SP1 was introduced with water delivered to that site before the rats' arrival. Rats initially found the new site by first searching unsuccessfully for water at the well-learned cued sites and then searching each well around the maze. After finding the new spatial goal, SP1 and LED-cued trials alternated such that, after completion of each LED-cued trial, the rat could run to the SP1 location and await a reward delivery; over the course of the first seven SP1 trials, the interval between arrival to the SP1 site and reward delivery ramped from 0.3 to $5 \mathrm{~s}$. Data collected at the new goal sites during "ramped training" trials were not included in neural or behavioral analysis. On day 2, rats were trained to alternate between SP1 and LED-cued locations for the shorter of 100 trials or $60 \mathrm{~min}$. On day 3 , the rats were given a block of 30 SP1 and LED-cued trials before SP2 was introduced. Between LED-cued trials, rats were required to first visit SP2 and then SP1, waiting at each location for $5 \mathrm{~s}$ before a reward was presented. On day 4, the rat continued to be trained on SP2-SP1 and LEDcued trials. Day 5 began with a block of 30 SP2-SP1 and LED-cued trials, before SP3 was introduced. Between LED-cued trials, rats were required to visit three spatial locations in the order SP3-SP2-SP1, waiting for $5 \mathrm{~s}$ to receive water at each place. Day 6 involved continued training on the three spatial locations along with the original LED-cued locations. After this sequence, rats were overtrained on the sequence of three positions for $1-12 \mathrm{~d}$ and then given at least $2 \mathrm{~d}$ on LED-cued trials only before being trained on a second sequence of spatial locations that never overlapped with LED-cued locations. One rat was also trained on a third sequence.

To assess the development of a schema as reflected in facilitation of new spatial sequence learning after initial sequence acquisition, we compared the latency to arrive at SP1 leading to a reward for trials on the first sequence to that for the second sequence over initial blocks of training trials (Fig. 1E). Analyses of learning focused on the trials after "ramped training" when rats were required to wait $5 \mathrm{~s}$ at the appropriate reward site.

Neural data with only SP1 and the cued sites are reported from 11 sessions from five rats. Data with SP2 and SP1 are reported from nine sessions with four rats, and neural data with SP1, SP2, and SP3 are reported from seven sessions from three rats. Data are reported from 15 learning sessions (SP1, SP2, or SP3) from five rats, seven sessions in which the new goal location is $1 \mathrm{~d}$ old (from four rats), three sessions when the new goal is $2 \mathrm{~d}$ old (from three rats), and 27 sessions in which the new goal is $8.34 \pm 3.61 \mathrm{~d}$ old (from three rats).

\section{Analyses}

The timestamps of unit action potentials, rat position, and reward delivery times were imported into MATLAB R2012b (MathWorks) for all analyses.

Behavioral events were distinguished as periods of waiting (WAIT) when the animal arrived at a goal and remained within $15 \mathrm{~cm}$ of the goal site for $5 \mathrm{~s}$ before, and not including, reward delivery and epochs of running (RUN) at velocity over $10 \mathrm{~cm} / \mathrm{s}$ within $4.2 \mathrm{~cm}$ of a goal site, excluding events $7 \mathrm{~s}$ before through $3 \mathrm{~s}$ after reward delivery.

Three spatial firing rate maps were constructed: (1) one for WAIT events, (2) one for clockwise RUN events, and (3) one for counterclockwise RUN events. Occupancy on the circle track was linearized and binned at $8.4 \mathrm{~cm}$ and not smoothed. Analyses were performed separately for rate maps in each running direction and then averaged for a single RUN metric. 


\begin{tabular}{|c|c|c|c|c|}
\hline $\begin{array}{c}\text { Cue } \\
\text { learning }\end{array}$ & $\begin{array}{c}\text { Critical test } \\
\text { week }\end{array}$ & & Extinction & Repeat \\
\hline $\begin{array}{l}\sim 1-10 \text { wks } \\
\text { LED-cued } \\
\sim \text { week } 4 \\
\text { drive implant }\end{array}$ & $\begin{array}{cc}\text { Day 1-2 } & \text { Day 3-4 } \\
\text { SP1 } & \text { SP2/SP1 } \\
\text { LED } & \text { LED } \\
& \text { (depicted) }\end{array}$ & $\begin{array}{l}\text { Day 5-6 } \\
\text { SP3/SP2 } \\
\text { SP1/LED }\end{array}$ & $\begin{array}{c}\text { Day } 7 \\
\text { no training } \\
\text { Day } 8-9 \\
\text { LED-cued }\end{array}$ & $\begin{array}{c}\text { Day 10-40 } \\
\text { same LED-cued } \\
\text { new SP } \\
\text { locations }\end{array}$ \\
\hline
\end{tabular}
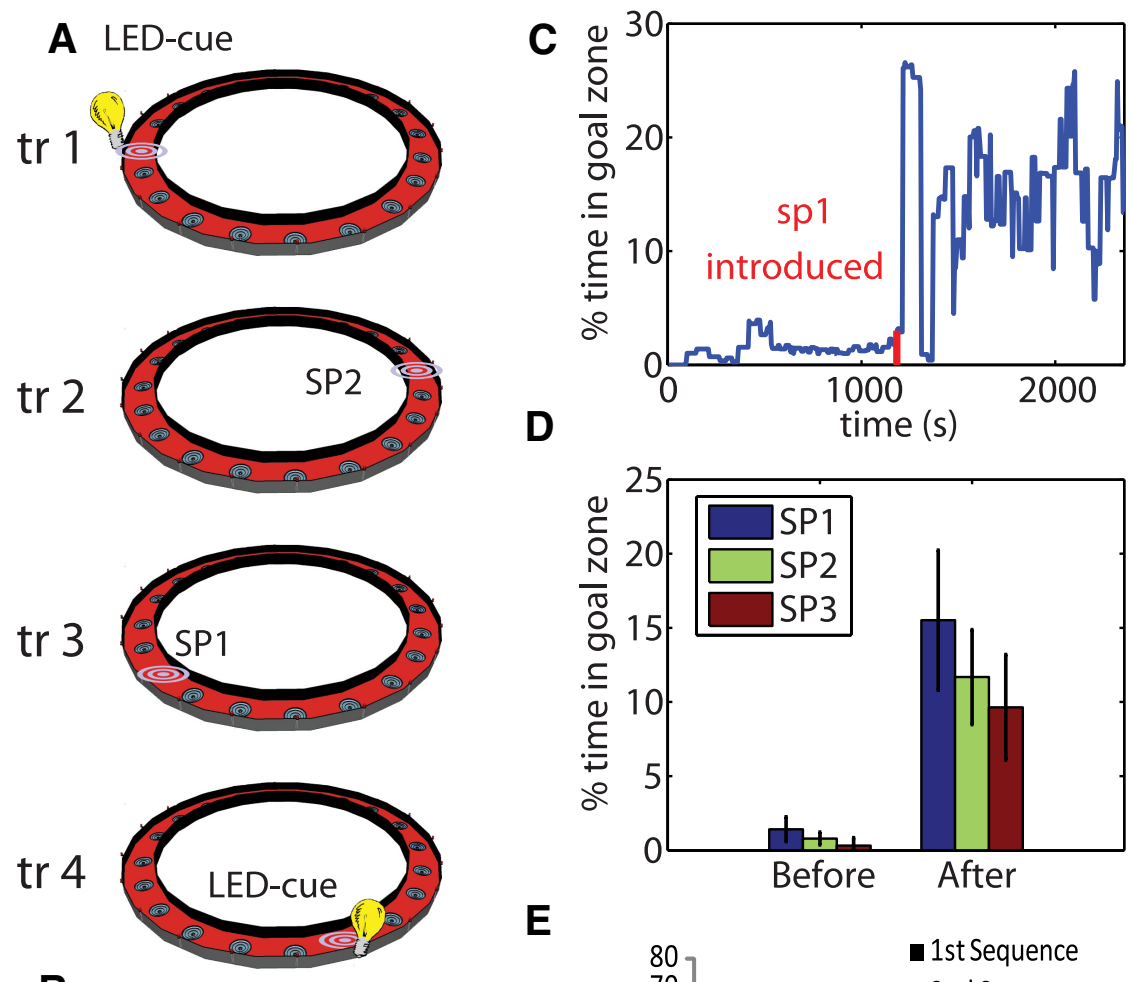

$\mathbf{E}$

B
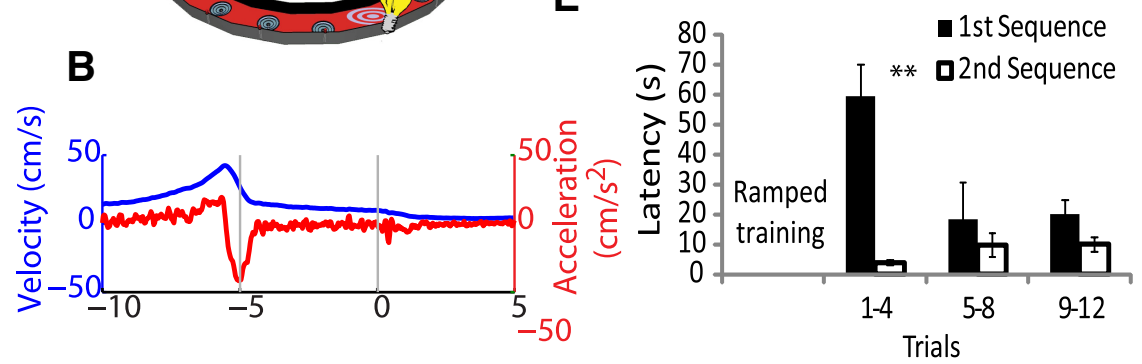

Figure 1. $\quad A$, The rat must visit the two spatial positions (SP1 and SP2) in order, waiting at each for $5 \mathrm{~s}$ for water reward. The next sequence begins with an LED-cued trial in one of four positions chosen pseudorandomly. $\boldsymbol{B}$, The average velocity and acceleration profile as the rat approached and waited at the goal sites. $\boldsymbol{C}$, The percentage of time a rat spent in the new spatial position not including reward consumption in a sliding 2 min window throughout an example session. Red tick marks indicated the time in which the new spatial location was first introduced. $\boldsymbol{D}$, The average percentage of time rats spent in the new spatial location before and after its introduction for all rats and for one, two, or three spatial goal locations (SP1, SP2, and SP3, respectively). $\boldsymbol{E}$, The latencies to arrive at SP1 for the first and second spatial sequence significantly differed in the early trials (trials $1-4$ ) at the full $5 \mathrm{~s}$ wait period, thus showing that learning one spatial sequence facilitated the learning of another. ${ }^{* *} p=0.01$.

Temporal analysis of WAIT epochs was based on perievent time histograms (PETHs) centered on reward delivery and binned at $250 \mathrm{~ms}$. We also defined cells as activated during WAIT events if a cell had an average firing rate above $2 \mathrm{~Hz}$ and $30 \%$ of the maximum firing rate around reward delivery in any $250 \mathrm{~ms}$ bin during the WAIT epoch $(-5$ to $0 \mathrm{~s}$ to reward delivery). The maximal firing rate was calculated \pm 20 s around reward delivery.

The LFP was taken from a channel with spiking units. Theta power was the power of the LFP bandpass filtered between 6 and $10 \mathrm{~Hz}$. Sharp-wave ripples were events that were 7 SDs higher in power than the average LFP filtered at $140-200 \mathrm{~Hz}$ with minimum duration of $20 \mathrm{~ms}$ and maximum duration of $150 \mathrm{~ms}$. The time of the ripple was the timestamp of the maximum waveform.
To probe the organization of the schema, we assumed that similar neuronal activity patterns observed when the rat is at different locations encode the common features of those events, whereas divergent firing patterns represent the distinct features of those events. The commonality and divergence of representations at multiple locations were assessed in six analyses that emphasize different aspects of similarity in neuronal firing patterns.

Cross-place activity. The similarity of temporal patterns of firing at multiple locations was measured by pairwise multiplication of the firing rate histograms $(-10$ to $+5 \mathrm{~s}$ centered on reward delivery and binned at $250 \mathrm{~ms}$ ) at each location (mean goals/session, 5.78; range, $4-8$ ), normalized by the maximal firing rate (see Figs. 2D, 6B,C). The average cross-place activity $(\overline{\mathrm{CPA}})$ was computed between every pair of locations for each cell during rewarded epochs:

$$
\overline{\mathrm{CPA}(t)}=\left\langle\frac{\lambda_{i}(t) \times \lambda_{j}(t)}{\lambda_{\max } \times \lambda_{\max }}\right\rangle,
$$

where $\lambda_{i}(t)$ is the trial-averaged firing rate at position $i$ at time $t$ relative to reward onset, $\lambda_{j}(t)$ is the firing rate at the equivalent time $t$ relative to reward onset at position $j$, and $\lambda_{\max }$ is the maximum average firing rate observed for that cell in any time bin at any location. Firing rates below a sliding threshold were then set to zero, driving the product to zero, and resulting in a metric of CPA over time that captures when high activity is present in two locations. With a threshold up to $5 \mathrm{~Hz}$, a significant increase in CPA was observed on arrival to the goal location.

Firing pattern selectivity index. The degree to which cells had different firing patterns across locations was measured with a selectivity index (SI) (Wirth et al., 2003; Komorowski et al., 2009):

$$
\mathrm{SI}(t)=\frac{n-\sum_{i=1}^{n}\left(\lambda_{i}(t) / \lambda_{\mathrm{pref}}(t)\right)}{n-1},
$$

where $n$ is the number of goal locations, $\lambda_{i}(t)$ is the trial-averaged firing rate at position $i$ at time $t$ relative to reward onset, and $\lambda_{\text {pref }}(t)$ is the maximal trial-averaged firing rate at any position at time $t$. SI values range from 1 , indicating activity at only one location, to 0 , indicating equivalent activity at all locations (see Fig. $3 A, B)$.

Spatial SI. Spatial selectivity was calculated based on each of the three firing rate maps. Unlike the firing pattern SI, $\lambda_{i}$ and $\lambda_{\text {pref }}$ did not vary over time and were based on the average firing rate recorded at the goal locations $(8.4 \mathrm{~cm}$ bin) from either the WAIT map (spatial SI WAIT) or the RUN maps (spatial SI RUN) (see Fig. 3E).

Temporal pattern ensemble correlation. Population vectors were composed of the mean firing rates for simultaneously recorded cells at each reward location at each time bin centered on reward delivery. The average similarity over time, $\overline{\operatorname{COR}(t)}$, was measured by calculating the Pearson's correlation coefficient of the population vectors, $X_{i}(t)$ and $X_{j}(t)$, at every time point, $t$, at pairs of locations, $i$ and $j$. Only ensembles of cells with more than eight units with a baseline rate $<4$ $\mathrm{Hz}$ were considered (22 ensembles; average \pm SD number of cells, 
$14.9 \pm 5.63$; range, $8-30)$. Low correlations indicate distinctive coding (see Fig. $3 F$ ):

$$
\overline{\operatorname{COR}(t)}=\left\langle\operatorname{corr}\left(X_{i}(t), X_{j}(t)\right)\right\rangle .
$$

Spatial ensemble correlation. As with the SI, ensemble correlations were also calculated using the same three spatial firing rate maps: the WAIT map and the two RUN maps. The spatial ensembles were constructed by average firing rates recorded at the goal locations $(8.4 \mathrm{~cm}$ bin) during either WAIT or RUN. The spatial ensemble correlation was calculated by taking the average Pearson's correlation coefficient of the spatial ensembles recorded at each goal site (see Fig. $6 D-F$ ).

Bayesian decoding. The extent to which firing patterns across locations differ should be reflected in accurate decoding. The spatial posterior distribution was calculated using the firing rates from both RUN and WAIT epochs using every other $500 \mathrm{~ms}$ block of spiking and positional data. Time was binned at $250 \mathrm{~ms}$, and the spatial firing rate map was binned as described above. Decoding accuracy was defined as the conditional probability of occupying the actual location given the observed spike counts for the time blocks not used to create the posterior distribution (Zhang et al., 1998) (see Fig. 4). Decoding was only applied to sessions with ensembles of cells of more than eight units with baseline rates $<4 \mathrm{~Hz}$.

Changes in firing patterns on the day of learning. To measure the extent to which neuronal firing patterns changed with learning, spatial firing rate maps were made for all periods when the rat's velocity was $>10 \mathrm{~cm} / \mathrm{s}$ before and after introduction of the new goal or for the first and second half of a session without new learning. Remapping was measured by Pearson's correlation of the before and after rate maps of each cell.

To measure learning-related changes in firing patterns during WAIT events, ensemble vectors of the average firing rates recorded at each WAIT site before and after learning were correlated (see Fig. $6 F$ ).

Changes in firing patterns over days. Two methods were used to study how cells encoded WAIT events across days. First, the spatial ensemble correlation was used to compare the new WAIT site to each of the welllearned, LED-cued sites. The average of these pairwise correlations was used as a metric for ensemble discrimination of the new WAIT event (see Fig. $6 E$ ). Second, we used a single-cell metric to analyze cells that were active at the new WAIT site on every day after learning. For these active cells, the average rate of a cell at the new site $(8.4 \mathrm{~cm}$ bin $)$ was subtracted from its rate at the other LED-cued WAIT sites. The minimum firing rate difference between the new and original sites was then calculated to measure the degree to which a new WAIT site was differentiated from any other WAIT site.

Principal component analyses. For visualization purposes, principal component analysis was used to reduce the $n$-dimensional (where $n$ is number of cells in the ensemble) representation of the goals. PETHs recorded on each trial were converted into $z$-scores based on overall firing rate means and SDs. The firing rates were then transformed into principal component space using the covariance of firing rates between neurons recorded \pm 20 s around goal delivery. For each goal location, the trial-averaged scores of the first two components are plotted as a function of time to reward (see Fig. 7B).

To show how the spatial representation of new and old WAIT events changed over days, firing rate maps for each cell were computed as described and then $z$-score normalized. Ensemble vectors were created using the average rates for each cell at each goal location. These vectors were then transformed into principal component space based on the covariance of the firing rates recorded across all locations. For each goal location, the first two components of the spatial ensemble representation are plotted (see Fig. 7C).

Significance testing. Metrics that were normally distributed were compared using ANOVAs and Student's $t$ test. However, most metrics were not normally distributed, and therefore, significance testing of means was done using Monte Carlo sampling with replacement with 10,000 repetitions (bootstrap). Changes from baseline were measured as the difference between the averages at every time point against a baseline calculated from the average -20 to +20 s around reward delivery.

\section{Results}

\section{Behavior}

Rats were trained on a task that required them to learn new goal sites in an environment with several well-learned goal locations (Fig. 1). Rats first learned to wait $5 \mathrm{~s}$ at one of several LED-cued locations to retrieve a water reward. Over the course of $6 \mathrm{~d}$, one to three new spatially defined goal sites were progressively introduced (SP1, SP2, SP3). Rats learned these new goal locations as reflected in significantly increased percentage of time spent in the rewarded area (not including reward consumption) after introduction of the reward (Fig. 1C,D; two-way ANOVA, main effect of time, $\left.F_{(1,24)}=91.55, p=11.6^{-9}\right)$.

Schemas are commonly characterized as facilitating new learning that is consistent with previous accumulated knowledge (Tse et al., 2007). Here, to examine whether initial acquisition of a spatial goal sequence facilitated subsequent learning of a second spatial goal sequence, we compared the latency to arrive at the first spatial goal (SP1) of the first learned spatial sequence to that for the second spatial goal sequence. As shown in Figure $1 E$, during initial spatial sequence acquisition, rats arrived in substantially longer latencies in first trials after the response requirement reached $5 \mathrm{~s}$ (see Materials and Methods), and thereafter latencies shortened. During learning of the second spatial sequence, unlike in the first sequence, latencies were initially low (two-way ANOVA, main effect of sequence, $F_{(1,3)}=19.93, p=$ 0.0004 ; time $\times$ sequence interaction, $F_{(2,3)}=0.0038, p=0.0038$; post hoc paired $t$ test for trials $1-4, t_{(3)}=5.11, p=0.014$ ) and remained low throughout testing. Savings in learning, as reflected by the significantly lower latencies during the second sequence, suggest that rats developed a schema for learning goal sequences that occurred within the familiar context.

\section{Hippocampal neurons activate during multiple well-learned WAIT events}

We first characterized how learned WAIT events are organized within a well-established schema of goal locations for which the rat has had at least $2 \mathrm{~d}$ of training. Within a well-established schema, it is expected that related memories will be both differentiated and linked via "nodes" that are characterized by similar representations of the common features between events. To investigate whether hippocampal networks contain a schema of WAIT events at multiple goal locations, we first asked whether the same behaviors at different locations were represented similarly by firing patterns of hippocampal neurons. Then, we also asked whether these WAIT representations also differentiated distinct memories of goal locations.

Several single neuron and ensemble analyses were adopted to characterize firing patterns of putative CA1 pyramidal cells ( $n=410$; average firing rate, $0.40 \pm 0.021 \mathrm{~Hz}$ ) through time and space. These analyses focused on WAIT events when rats stopped at the goal loci before reward delivery and RUN epochs when rats ran through the locations and no reward was available. WAIT epochs allowed us to assess firing associated with anticipatory behavior at the goal location, whereas RUN epochs provide a baseline of spatial firing without the anticipatory behavior.

Examination of firing patterns before and during WAIT events revealed that many cells fired at multiple goal locations (Fig. 2A). For example, Cell 1 in Figure $2 A$ had two firing fields around the spatially separate goals sites 1 and 12 and did not fire at other goal locations, whereas Cell 2 fired at goals 1, 12, and 17. An analysis of the number of locations where activation occurred during WAIT events revealed that $40 \%$ of neurons were active 

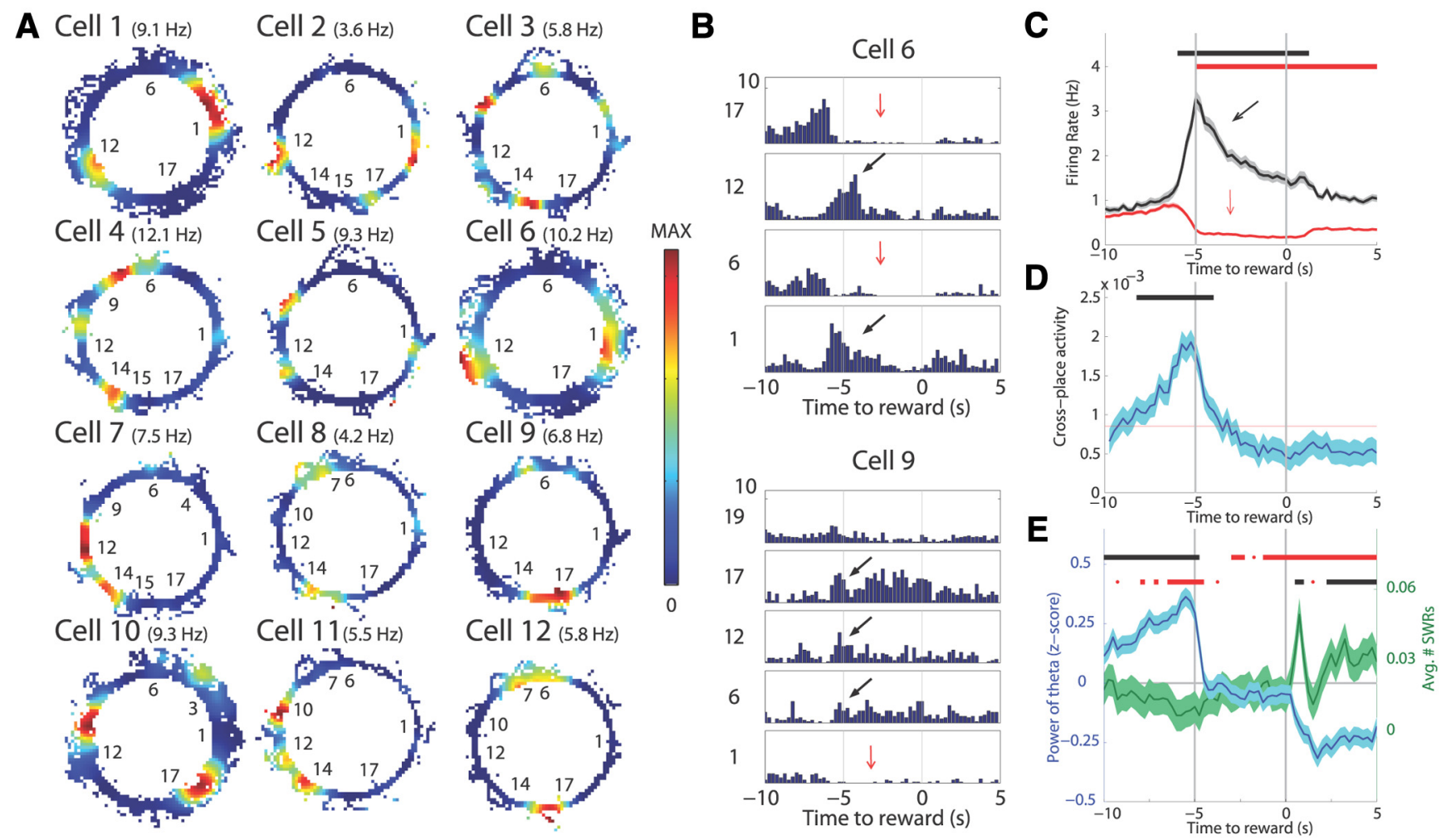

Figure 2. A, Example rate maps (both RUN and WAIT events) for 12 cells that fired at multiple goal locations (numbered). The color for each rate map is scaled to the maximum firing rate of each cell reported in parentheses by the cell label. $\boldsymbol{B}$, Histograms from two cells centered on reward delivery at $t=0 \mathrm{~s}$. Each block is a different reward site. The gray bars indicate the $5 \mathrm{~s}$ the rat waited for reward. Black arrows show burst of firing on arrival, and red arrows show slight decrease in baseline firing rate as rats wait for reward. $C$, Mean cell firing rate during active WAIT event (black) and during non-active WAIT event (red). Black bar is significant firing above baseline $(p<0.05)$ for the active cells, and red bar is significant firing below baseline for the non-active cells. $\boldsymbol{D}$, The CPA was calculated as the product of the normalized firing rate of a cell during approach to two goal locations. On average, cells were more likely to be active in multiple locations during the beginning of the WAIT period. $\boldsymbol{E}$, The power of theta decreases on arrival to the goal site and further still after reward consumption. In contrast, the number of sharp-wave ripples increased only after reward consumption, indicating that the burst of activity on arrival to the goal is not attributable to a ripple event. Black bars show data above baseline, and red bars represent data below. The top error bars show significance testing for theta power, and the bars underneath correspond to analysis of sharp-wave ripples.

during WAIT events at one location, $15.6 \%$ at two locations, and $9.8 \%$ at more than two goal locations, indicating that $39 \%$ of the neurons that were active during at least one WAIT event fired during more than one WAIT event.

Firing associated with WAIT events was not uniform in time such that, on arrival to a subset of goal areas, neurons often released a burst of spikes, followed by either a period of decaying activity or silence (Fig. $2 B, C$ ). This firing pattern can be observed clearly in the firing behavior of Cell 6 in Figure $2 B$, which increased firing rate on arrival to locations 1 and 12 and decreased firing at the other two locations. Comparing the average response from all cells that activated during WAIT events versus those that did not, activation during WAIT events was characterized by an increase in firing rate on arrival ( $5 \mathrm{~s}$ before water delivery) to the goal site, followed by a slowly tapering level of firing during WAIT events. In contrast, cells that did not exceed the activation threshold at WAIT locations decreased firing rates from baseline (Fig. 2C).

To examine the similarity of activation patterns across locations, we measured CPA between all pairwise combinations of WAIT events at well-learned sites for all cells. This analysis revealed a common activity peak at the beginning of WAIT events, followed by diminished CPA during the course of the ensuing WAIT event (Fig. 2D). The CPA increase at the outset of WAIT events was observed even after setting rates below a sliding threshold to zero (see Materials and Methods) to measure only time points of high activity in both goal locations.
The activation of cells at the goal site coincided with the rat decelerating (Fig. 1B), thus possibly confounding a change in neural activity attributable to reduction in running speed with firing that encodes the WAIT event per se. The firing rate of hippocampal cells is generally positively correlated with running speed (McNaughton et al., 1983; Geisler et al., 2007), except for the brief bursts of activity during sharp-wave ripples when the animal is typically not moving (Buzsáki et al., 1983; O’Neill et al., 2006). Here we also observed a positive correlation between running speed and population firing rate in the $20 \mathrm{~s}$ before arrival to goal sites $\left(r=0.24, p=4.9^{-167}\right)$ and a weak but significant positive correlation between acceleration and population rate during the same epoch $(r=0.02$, $p=0.01)$. In contrast, during WAIT epochs, population firing rate was negatively correlated with acceleration $(r=-0.11$, $\left.p=5.7^{-15}\right)$, although the positive correlation with velocity was maintained $\left(r=0.25, p=4.35^{-70}\right)$. Most importantly, although the velocity and acceleration profiles were very similar at all goal sites, cells were never active at all the goal locations. Also, the probability of observing sharp-wave ripples was lowest on arrival to the goal sites (Fig. 2E). Combined, these observations show that the burst of activity on arrival to the goals cannot be explained simply by changes in running speed, acceleration, or spiking in a sharp-wave burst, suggesting that they likely reflect neural processing related to behavior during the WAIT events. 

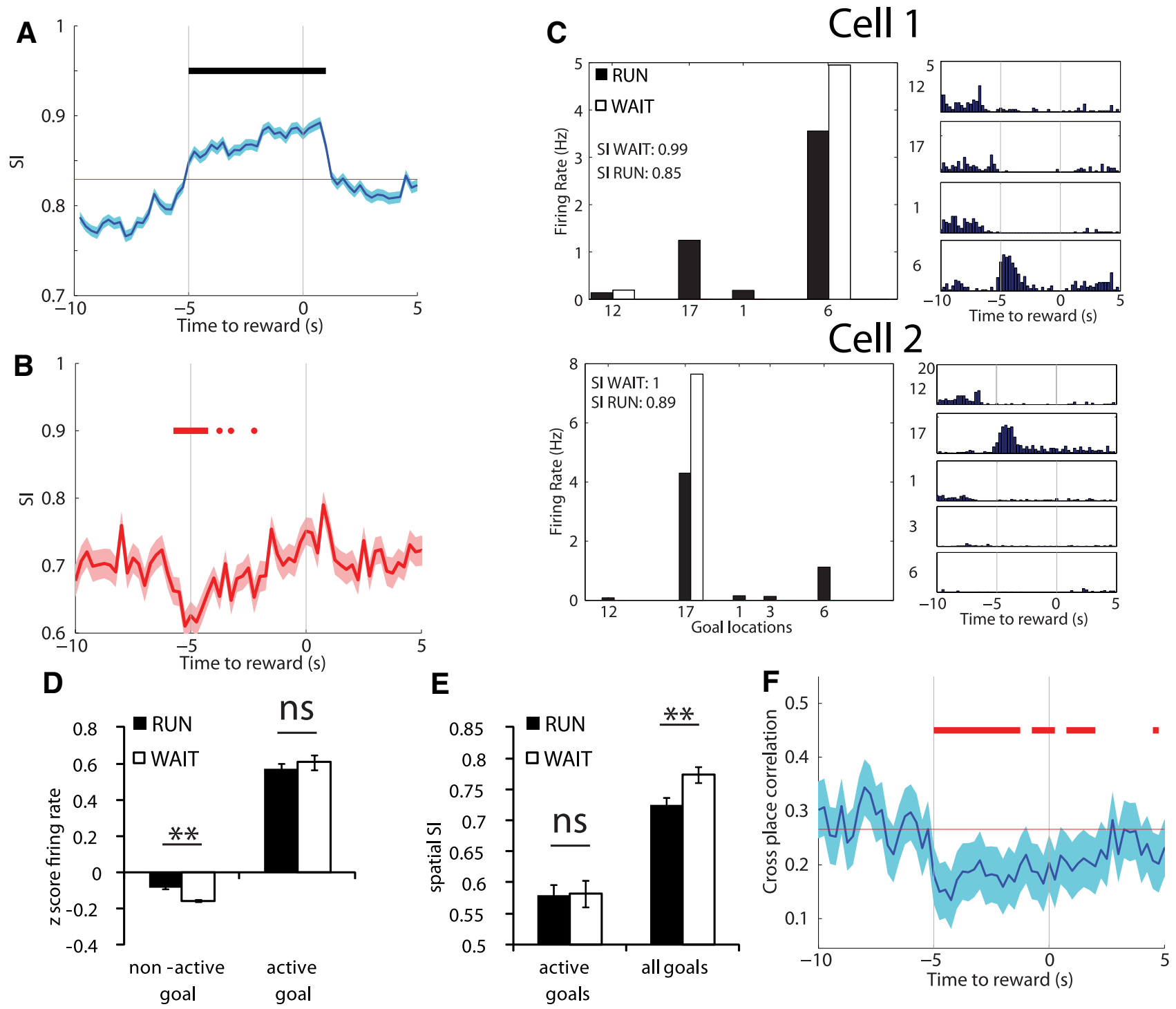

Figure 3. $A$, The average of the Sl of cells as measured by the PETH recorded at each WAIT location. The Sl increased on arrival to the goal site $(t=-5 \mathrm{~s})$ and remained high until just after reward delivery. $\boldsymbol{B}$, The same selectivity metric but calculated only for cells that were active at multiple sites. The $\mathrm{SI}$ for only the sites in which a cell was active decreased on arrival to the goal sites during the same period of high CPA. C, The firing rates of two cells at each WAIT site as calculated from the firing rate map (left) and the PETH (right). During WAIT epochs, the firing rate is more selective to the preferred goal location. $\boldsymbol{D}$, The average $z$-score firing rate as calculated from the firing rate map is higher during RUN epochs at sites in which the cell does not activate during WAIT epochs, whereas there is no difference in rate at the sites for which a cell is active during the WAIT epoch. $\boldsymbol{E}$, For cells that are active for multiple WAIT epochs, the selectivity across the active sites is equally poor during WAIT and RUN epochs, although across all goal locations, selectivity is higher during WAIT epochs, indicating that cells are selective during WAIT epochs attributable to decreases in firing rate in locations in which they were active during RUNs. $\boldsymbol{F}$, The ensemble correlation decreased on arrival to the goal location, indicating more distinct neural representations. Red bars show significant (bootstrap, $p<0.05$ ) epochs below baseline, and black lines show epochs above baseline. ${ }^{* *} p<0.01$.

\section{Hippocampal activity distinguishes well-learned WAIT events at different locations}

In addition to relating memories by their common features, schemas must distinguish memories by differences in their features. There are potentially many ways by which hippocampal ensembles could discriminate memories. A simple mechanism for generating a high degree of memory separation would be for cells to activate during a single event and remain silent for others. Another possibility is that cells distinguish events based on firing rate, and previous studies have shown that rate differences could allow for comparable discrimination as the simpler, binary coding scheme (Leutgeb et al., 2005). Finally, it is possible that, although single cells may discriminate a subset of events poorly, on the ensemble level those events are well discriminated because of the contribution of other cells that are selective to that subset. We explored whether any of these three coding scenarios could describe how cells distinguish goal sites from one another.

Although many neurons were active at the outset of multiple WAIT events, these events could be potentially discriminated by firing rate and differing temporal patterns of activity. For example, Cell 6 depicted in Figure $2 B$ fired during WAIT events in two locations, with different firing rates at different times and decreased firing rate at the other two locations. To evaluate the extent to which WAIT events at different locations were associated with distinct temporal firing patterns, we calculated the SI for each cell $(n=410)$ during WAIT events at all well-learned goal locations. There was a prominent increase in SI that began on arrival at the goal and persisted throughout the $5 \mathrm{~s}$ WAIT period, indicating that single neurons highly discriminated those events at different locations (Fig. $3 A$ ). When these analyses were 
restricted to cells that were active in multiple locations $(n=164)$, there was a drop in selectivity for the locations in which a cell showed CPA (Fig. 3B). This drop in SI can be seen in Figure $3 B$ on arrival to the goal site, the same time at which cells showed high CPA. Although many cells discriminated WAIT events by being active only at a single site, other cells encoded a subset of the WAIT events similarly by increasing firing rate on the outset of the events.

Although the SI analysis can capture the temporal dynamics as rats remain within the $15 \mathrm{~cm}$ of the goal site, this time series analysis is potentially confounded by differences in occupancy of specific locations; so, firing rates at the goal locations were additionally analyzed based on the average firing rates, as calculated from the spatial rate map, at goal locations during WAIT and RUN events. As can be seen in the examples in Figure 3C, cells showed different firing rates during WAIT and RUN epochs through the same goal locations. In the example shown in Figure $3 C$, Cell 1 fired in locations 6 and 17 during RUN epochs but during WAIT epochs only fired in location 6 and was virtually silent in the other locations, as demonstrated by the low firing rates in locations 1,12 , and 17 during the WAIT event in the adjacent histogram. In both examples, the cells fired in more goal locations during RUN events. The difference in firing rate during RUN epochs was not a general increase in drive to the cells because there was no difference in firing rate during WAIT epochs (mean rate, $2.30 \pm 0.13 \mathrm{~Hz}$ ) compared with the RUN epochs (mean rate, $2.20 \pm 0.11 \mathrm{~Hz}$ ) through the goal location in which a cell was active (bootstrap, $p=0.56$ ) (Fig. 3D). In goal locations in which a cell was not active during WAIT events, firing rates increased during RUN events (mean rate WAIT, $0.30 \pm 0.03 \mathrm{~Hz}$; mean rate RUN, $0.49 \pm 0.03 \mathrm{~Hz}$; bootstrap, $p<0.0001$ ) (Fig. $3 D)$. It is important to note that cells never fired at all goal locations during WAIT events despite identical behavioral demands. By definition, locations in which a cell was active were associated with significantly higher average firing rates than locations in which a cell was not active (bootstrap, $p<0.0001$ ).

As suggested by these rate differences, hippocampal neurons discriminated locations during WAIT events (spatial SI, $0.83 \pm$ 0.01 ) better than during RUN events (spatial SI, $0.79 \pm 0.01$ ) through the same locations (bootstrap, $p=0.039$ ). This difference in spatial discrimination during WAIT and RUN epochs was more pronounced in the cells that were active in multiple locations (spatial SI WAIT, $0.77 \pm 0.01$; spatial SI RUN, $0.72 \pm 0.01$; bootstrap, $p=0.009$ ) (Fig. $3 E$ ). However, for the same cells, there was no difference in spatial SI when the analysis was restricted to only the subset of goals in which a cell was active (spatial SI WAIT, $0.58 \pm 0.02$; spatial SI RUN, $0.58 \pm 0.02$; bootstrap, $p=0$ 0.93 ) (Fig. 3E). Therefore, single cells better discriminated goal locations during WAIT events by limiting the number of events for which they were active and not by increasing rate differences in the subset of active locations.

Given that many cells fire at multiple locations, we tested the extent to which the pattern of population activity distinguished WAIT events at different locations. As rats approached the reward site and waited for the water reward, the pairwise correlation of ensemble activity of all putative pyramidal cells recorded at well-learned goal locations decreased on arrival and remained low throughout the $5 \mathrm{~s}$ WAIT epochs (Fig. $3 F$ ). Therefore, as the rat arrived at different goal locations, there was a rapid change in hippocampal activity that resulted in the associated neural ensembles becoming less similar. Spatial ensemble correlation also indicated that goal locations were less correlated during WAIT epochs (spatial ensemble correlation, $0.176 \pm 0.02$ ) than RUN events (spatial ensemble correlation, $0.22 \pm 0.02$ ) through the same location (bootstrap, $p=0.044$ ). These findings show that, although a subset of cells showed common activity in a subset of WAIT locations, the ensemble of neurons discriminated WAIT events throughout the entire $5 \mathrm{~s}$ WAIT period.

The analyses above relied on averaging firing rates over space or over time and only considered how distinct goal locations were from one another rather than being globally distinguished from other maze locations. To estimate the overall discrimination of WAIT events at each moment in time, we examined Bayesian decoding of neural activity for the locations of different WAIT events. The best decoding accuracy was observed at the beginning of the WAIT epochs when there was the highest probability of the rat occupying its actual location based on ensemble activity (Fig. $4 A$ ). Consistent with our results on high spatial selectivity and low ensemble correlation during WAIT epochs, the beginning of WAIT events was also associated with low probability of the rat occupying the other goal locations spatially separate from the rats' actual location (Fig. 4B). In addition, the overall uncertainty of the rats' location (Shannon's entropy) was lowest during the beginning of the WAIT epochs (Fig. 4C), showing that the rat was least likely to be in any other location other than its actual location during WAIT events. Therefore, despite the subset of cells that fired in multiple goal locations, on the ensemble level, WAIT events were well discriminated from one another and from locations elsewhere on the track.

\section{Hippocampal coding of new and previously learned WAIT events changes over days}

We also explored characteristics of firing patterns before new learning that predict the assimilation of representations of new WAIT events with learning. Based on the property of schemas by which new learning is accelerated for material that is most consistent with existing schema structure (Bartlett, 1932; Tse et al., 2007), we hypothesized that cells that begin to fire during WAIT events with learning would be the same cells that already fired during WAIT events before new learning.

To examine this prediction, we introduced a new, spatially defined goal location midway into a recording session to determine how cells change firing at the new and old goal locations with learning. We distinguished cells ( $n=44$ of 192) that were activated (see Materials and Methods) during the WAIT event at the new goal site from those that fell below this activation threshold (Fig. 5, Cells 1-3). These cells showed clear activity at the new WAIT location but also at previously learned WAIT locations (Fig. 5, Cell 2 in location 6).

The average response during the WAIT event of all cells that were active at the new site was a strong initial burst of activity on arrival to the site, followed by a decrease in average firing rate as cells that were active stopped firing (Fig. 6A). Also, cells that were not active during new WAIT events showed a slight decrease in firing rate during the WAIT event as can be seen by the lowerthan-baseline firing rate starting $5 \mathrm{~s}$ before reward delivery (Fig. $6 A)$.

Before the new goal site was introduced, $91.3 \%$ of cells that would become active at the new goal site were active at the previously learned WAIT locations. These active cells also fired at more of the previously learned WAIT locations $(2.26 \pm 0.22$ sites) than those cells that were not active at the new site (1.39 \pm 0.07 sites; bootstrap, $p<0.0001$ ) (Fig. $6 B$, inset). CPA analysis showed that this activity common to multiple locations occurred as rats arrived to the goal locations (Fig. $6 B$ ). These results suggest 

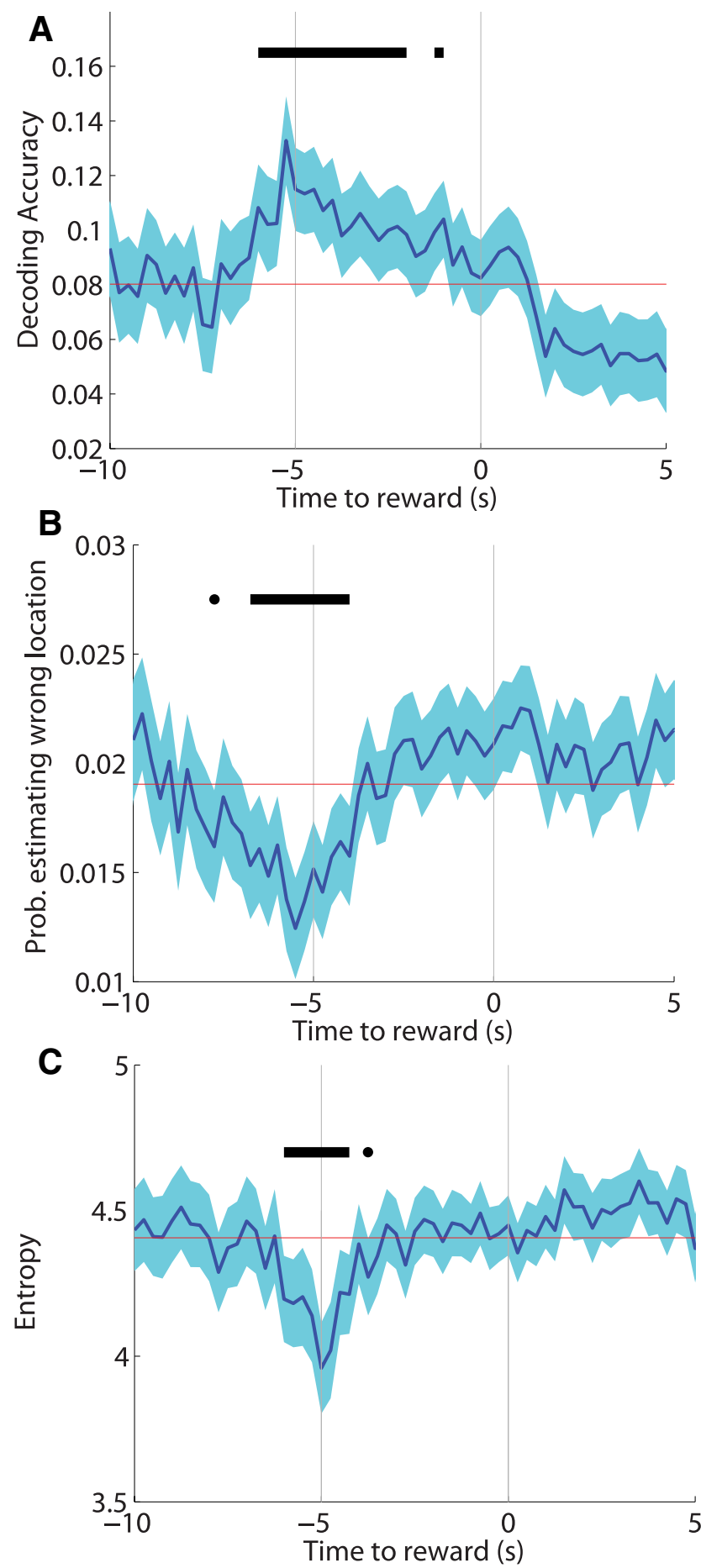

Figure 4. A, Bayesian decoding of hippocampal spiking data revealed the highest probability of the rat occupying its actual location on arrival to the WAIT site. Also during arrival, the probability of decoding the rats' position to the wrong location $(\boldsymbol{B})$ and the overall uncertainty of the rats' location ( $\boldsymbol{C}$ (Shannon's entropy) were less than baseline (red line). Black bars indicate significant $(p<0.05)$ deviations from baseline.

that the networks that encoded the new goal locations were those that were active during other WAIT events.

After introduction of the new WAIT site, cells that were active at the new WAIT site also showed activity at previously learned WAIT sites (mean number of sites, $2.29 \pm 0.20$ ) as reflected in a high CPA between the new goal location and the other goal locations upon arrival (Fig. 6C). To determine whether the high CPA between new and original locations was associated with similar ensemble activity at new and original WAIT events, spatial ensemble correlations ( 10 ensembles with $16.0 \pm 1.25$ cells) were calculated in a pairwise manner between the new WAIT site and original WAIT sites before and after learning (52 pairs total). These analyses showed that, before learning, activity during RUNs through the to-be-rewarded site was poorly correlated $(r=0.10 \pm 0.04)$ with activity during RUNs through the other goal sites (Fig. 6D). However, after the introduction of the new WAIT site, the ensemble activity at the new site became more correlated with activity at the previously learned goals during both RUN $(r=0.32 \pm 0.04, p<0.0001)$ and WAIT $(r=0.33 \pm$ $0.08, p=0.003$ ) events (Fig. $6 D$ ). This high correlation between the new WAIT site and previously learned WAIT sites was in contrast to the low ensemble correlations restricted $(r=0.14 \pm$ 0.04 ) to the previously learned WAIT events (bootstrap, $p=$ 0.02 ) (Fig. 6D).

To address at what point activity at the new WAIT site became decorrelated with activity at other WAIT sites, we tracked activity during WAIT events in the days after learning. Activity at the new WAIT site was only compared with the overtrained LED-cued sites to see how new information was incorporated into the wellestablished schema. On the first day of learning, the ensemble activity at the new WAIT site showed the highest correlation with activity at the LED-cued sites (Fig. 6E). Over the course of days, the spatial ensemble correlation between the new WAIT site and the overtrained LED-cued sites decreased, such that there was a significant correlation between time since learning and the average spatial ensemble correlation between new and original WAIT sites $(r=-0.45, p=0.009)$.

High average ensemble correlations between new and original WAIT sites were thought to be attributable to single cells that fired at a similar rate at the new WAIT and at one or more of the original WAIT sites. As suggested by the change in ensemble correlation, the smallest rate difference between the new and original sites was observed on the day of learning (rate, $1.73 \pm 0.49 \mathrm{~Hz}$ ) and increased over the course of days, giving rise to a significant correlation between minimum rate difference and days after learning (see Materials and Methods) $(r=0.42, p=0.02)$.

The pair of locations that produced the minimum rate difference was not necessarily the closest two locations in actual space because there was no correlation between the distance separating the goal sites and minimum rate difference $(r=0.12, p=0.53)$. Furthermore, there was no correlation in the total amount of distance traveled during the WAIT epoch and the number of days since learning $(r=-0.14, p=0.36)$, suggesting that there was no systematic change in behavior at the new WAIT site in the days after learning.

These data suggest that integrating the new goal location into the preexisting schema involved cells initially generalizing the original WAIT events to the new WAIT event, but over extended time, the full set of goal locations became well distinguished by hippocampal cell activity.

\section{Learning induces modification of preexisting schemas to accommodate new memories}

After characterizing the dynamics of the schema for previously acquired goal locations, we next analyzed how an established schema changes to assimilate new WAIT events. We observed that learning a new goal location caused a remapping of spatial firing patterns as shown by a lower correlation of the spatial firing rate maps before and after learning $(r=0.44 \pm 0.02)$ than the 


\section{Before New Goal}

\section{After New Goal}
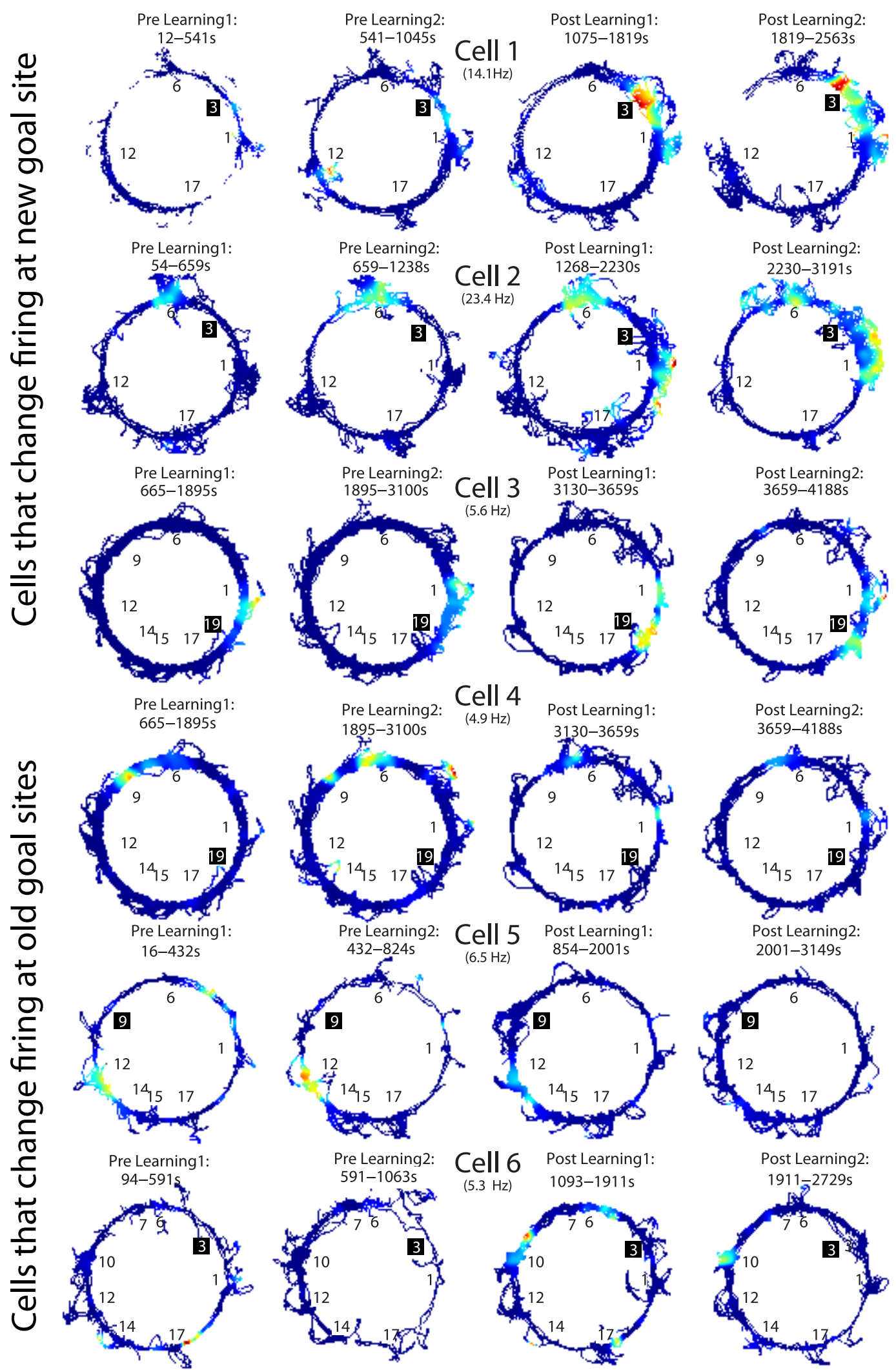

Figure 5. Example firing rate maps of six cells that changed firing rates at either the new or old WAIT locations. The black numbers indicate the old goal locations, and the white text and black background represent the location of the new goal. Rate maps constructed from both WAIT and RUN epochs. Cells that fired at the new WAIT locations also tended to fire at original WAIT locations before learning (e.g., Cell 2 in location 6) and/or after learning (e.g., Cell 3 in location 1). Cells also changed firing rate at previously learned goal locations, including decreases in firing rate (e.g., Cell 4 in locations 6 and 9 ) and increases in firing rate (e.g., Cell 6 in location 10). The color scale for each cell is scaled such that red is the maximum firing rate (reported in parentheses) observed before or after learning. 

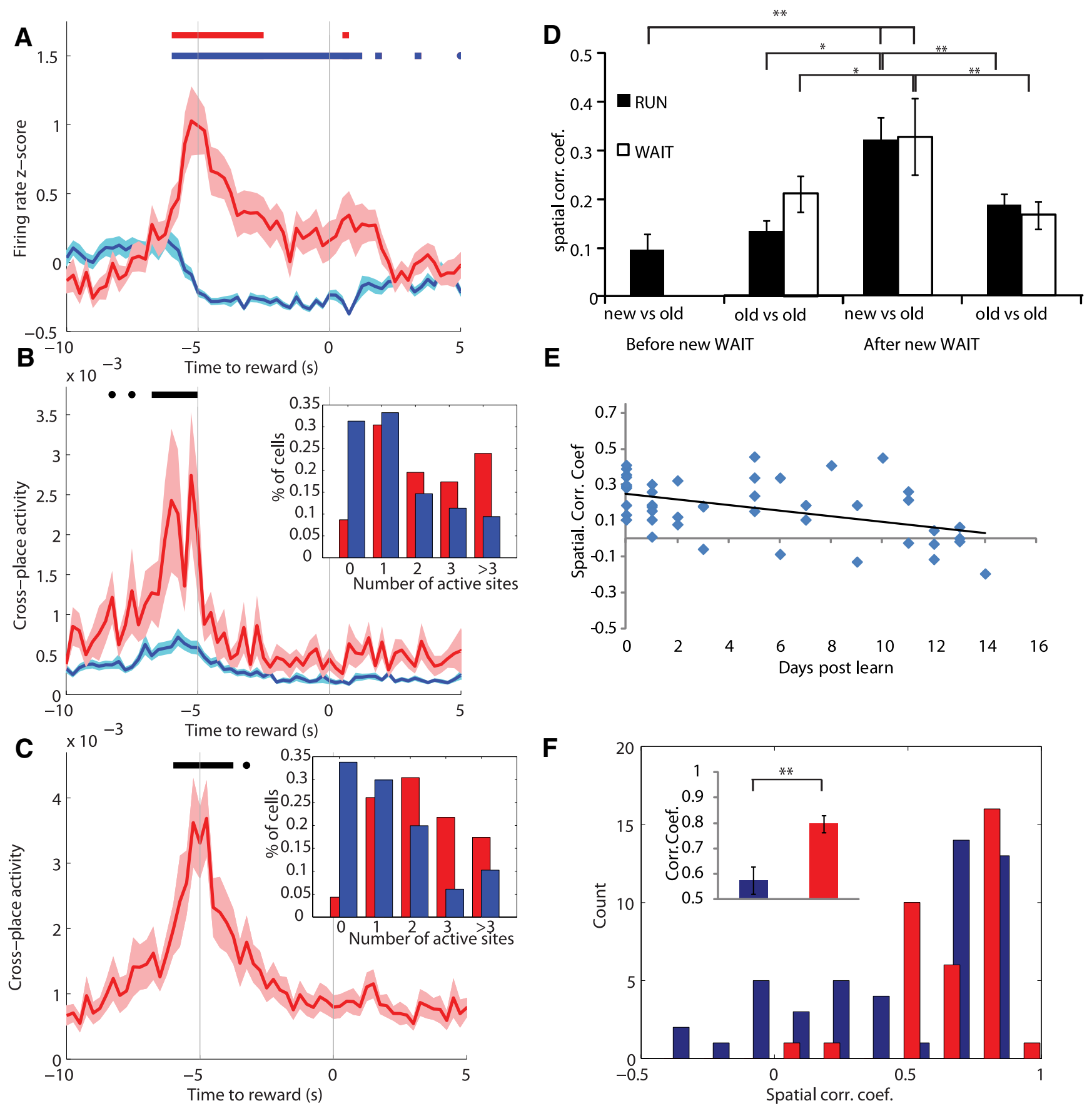

Figure 6. A, The average firing rate at the new WAIT site for cells that were active and those that were not (blue). Red bar shows epochs of firing rate significantly above baseline for the active cells, and the blue bar is epochs below baseline for non-active cells. $\boldsymbol{B}$, Cells that were active at the new goal site (red) fired on arrival to multiple WAIT events before the introduction of the new goal site, as can be seen by the high CPA. Black bar shows epochs in which the CPA before learning is above baseline and higher for cells active at the new goal site compared with the CPA for cells not active at the new goal site (blue). Inset, Distribution of the number of sites in which cells (red, active at new site; blue, not active at new site) were active before the introduction of the new site. C, Cells that fired at the new WAIT location fired at original WAIT locations after learning as seen by a high CPA between original and new sites. Inset, Distribution of the number of sites in which cells (red, active at new site; blue, not active at new site) were active after the introduction of the new site. $\boldsymbol{D}$, Before introduction of the new WAIT site, ensemble activity at the goal sites was poorly correlated (new vs old). After the new WAIT site was introduced, ensemble activity at the new site became more correlated with activity at the previously learned goal sites (new vs old). In contrast, ensemble correlation at the original goal locations remained low throughout learning (old vs old). $\boldsymbol{E}$, In the days after the introduction of a new WAIT site, the average ensemble correlation between that location and the well-learned LED-cued location decreased, indicating increased distinctiveness of the WAIT event representation over time. $\boldsymbol{F}$, The distribution of ensemble correlations of the overtrained LED-cued sites for the first and second half of a recording session on learning days (blue) and days with only LED locations (red). The distributions significantly differed, and the representation of the well-learned locations changed more on learning days as shown by lower average ensemble correlations (inset). ${ }^{*} p<0.05$, ${ }^{* *} p<0.01$.

correlation of rate maps of the first and second half of a session without new learning $(r=0.53 \pm 0.02$; bootstrap, $p=0.002)$. Rate map correlations did not differ between the learning sessions for the first $(r=0.45 \pm 0.04)$, second $(r=0.45 \pm 0.04)$, and third $(r=0.43 \pm 0.04)$ goal locations (all $p>0.19)$, and the rate map correlations for each of these three types of learning session were lower than the rate map correlations on days without learning (bootstrap, all $p<0.05$ ). These results indicate that each new learning event caused an equivalent extent of reorganization in hippocampal networks. 


\section{A Actual Layout}

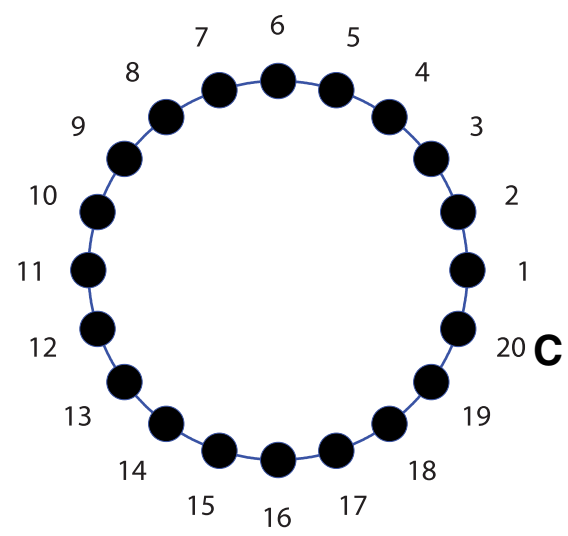

B

Time to reward: $-6 \mathrm{~s}$
Hippocampal Schema

$\begin{array}{cccc}-5 \mathrm{~s} & -2.5 \mathrm{~s} & 0 \mathrm{~s} & +1 \mathrm{~s} \\ \text { (arrival) } & 17 & & \text { (reward) }\end{array}$
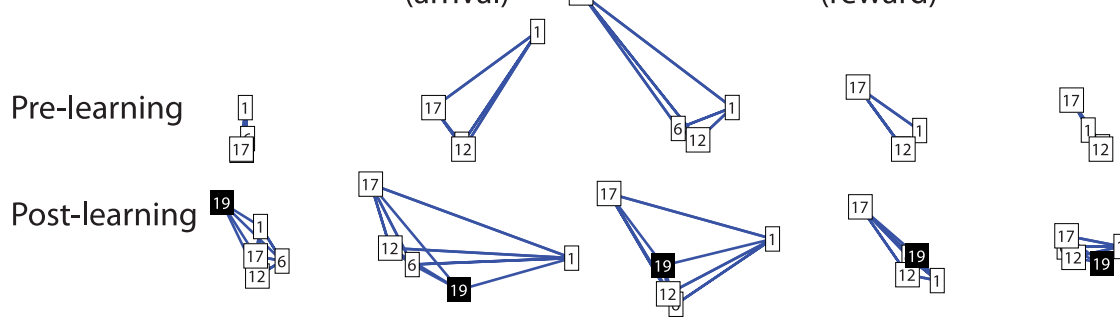

Days

post-learning: Day 0

Day 1

Day 5

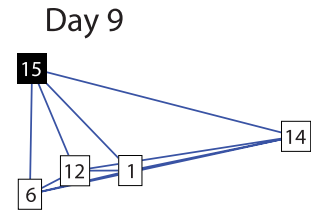

Figure 7. A, The circle track consisted of 20 possible water ports $22 \mathrm{~cm}$ apart of which four to eight ports could be rewarded during a single session. $\boldsymbol{B}$, The number boxes are the identity of rewarded water ports, and the new goal site is depicted in black with white text. The ensemble representations of the WAIT events are plotted by the first two principal components at each time relative to reward. The top row is the prelearning schema, and the bottom row is the postlearning schema with the addition of the new goal location plotted in the same principal component space. At arrival ( $-5 \mathrm{~s}$ to reward), the representations of the goal locations became less correlated because of the fact that the ensemble representation of the goal locations occupy different regions of hippocampal state space. In the postlearning schema, preexisting locations are represented differently by the same ensemble, but original WAIT events are still well discriminated by the hippocampal network. C, Immediately after learning, the new WAIT site is represented similarly to preexisting WAIT sites, as visualized by the new and original WAIT sites occupying a similar region of principal component space. Over the course of days, the new WAIT site is represented differently from the original WAIT sites as seen by an increase in the distance between the new WAIT site to the old. The data are plotted from different sessions and different rats to clearly depict the trend described in Figure $6 E$.

More specific to theories of schema modification, it is expected that the assimilation of new information causes changes in the preexisting schema to accommodate new memories, and this should be reflected in substantial changes in firing rates during the WAIT events. To calculate how the representation of a goal site changed over the course of a session, the spatial ensemble correlation was calculated between the first and second half of the recording sessions. Low correlations indicate a change in the representation of the WAIT sites. On days with only LED-cued locations, the majority of WAIT events had a stable representation as indicated by the high average spatial correlation between the first and second half of the session (Fig. $6 F$ ). In contrast, on days with learning, some sites maintained a stable representation throughout learning (Fig. 5, Cell 2 in location 6), whereas other locations were associated with cells that changed their firing rates as the new goal location was learned (Fig. 5, Cell 4 in location 6, Cell 6 in location 10). The learning of the new goal site was accommodated by changes in firing rate in at least a subset of the original goal locations as seen by a lower average spatial correlation between the first and second half of sessions with learning $(r=0.57 \pm 0.05)$ versus days without $(r=0.79 \pm 0.03$; bootstrap, $p=0.002)$ and a significantly different distribution of correlations for days with and without learning (Kolmogorov-Smirnov statistic $=0.38, p=0.004)($ Fig. $6 F)$.

\section{Schemas are dynamic during task performance and across days}

We examined the structure of neural ensemble representations of the WAIT events at different locations (Fig. 7A) in terms of projections onto the first two principal components of the ensemble (see Materials and Methods) (Fig. 7B,C). This analysis revealed that hippocampal schemas of WAIT events were dynamic in two ways. First, during stable performance, as the rat traversed the maze and arrived at the goal, there was a rapid distortion of the schema (Fig. 7B). On arrival and as the rat awaited reward, the WAIT events were represented in different regions in hippocampal state space, thus increasing the spatial selectivity of the cells and decreasing ensemble correlation between locations. Thereafter, the representations of WAIT events converged.

Second, on learning a new WAIT location, the existing schema accommodated during learning to assimilate the new WAIT events. The WAIT events in the updated schema were represented in a different part of hippocampal state space, but as before, the preexisting goals were well distinguished (Fig. $7 B$ ). The new WAIT events in the updated schema were initially represented similarly to the preexisting WAIT events. Over the course of days, the new WAIT location became well distinguished from the old, as visualized by an increase in the distance between the new and original goal locations in the principal component space (Fig. 7C).

\section{Discussion}

Here, we begin to explore the neural substrate of a hippocampal schema as reflected by network activity that links and distinguishes reward-anticipatory behaviors at different locations in an environment, integrates new memories to preexisting schemas, and modifies the preexisting schema to accommodate additional memories. Bartlett (1932) emphasized that new information that is consistent with preexisting knowledge is readily assimilated within existing schemas. Piaget (1929) acknowledged that the assimilation of new memories would usually require updating the schema to accommodate new information. Under this framework, Tse et al. (2007) demonstrated the essential role of the hippocampus in the assimilation of new memories into a preexisting schema for the locations of food rewards.

We observed that incorporating a new goal location into a preexisting schema of goal locations changed the hippocampal network in several ways. First, cells that fired at original goals 
began to fire at the new goal site in addition to firing at the previously learned goal sites. The common activity across the new and original goal locations diminished the discriminability of the new goal location by hippocampal cell firing. Only in the days after learning did the representation of the new goal location become decorrelated from those recorded at the original goal locations. We also observed that firing at the original goal sites changed as the new goal location was learned. Despite the plasticity that occurred as new goal information was accommodated, the ensemble continued to discriminate the well-learned goals during reward anticipation.

Several previous studies have reported that changing a goal location does not influence place cell firing (Speakman and O'Keefe, 1990; Trullier et al., 1999) whereas others report shifts in the mapping of goal locations (Gothard et al., 1996; Kobayashi et al., 1997), and more recent studies have shown that there may be an overrepresentation of goal areas in a water-maze task (Hollup et al., 2001) and in paradigms that require association of reward and location (Dupret et al., 2010). However, in all of these studies, goal locations were moved, rather than added as in the current study, and were aimed at examining the influence of the goal event on spatial mapping. By exploring the addition of goal locations to a preexisting and continuing representation of goal events, we are able to observe the evolution of a schema for the same behavior at multiple goal sites within an environment.

Our findings that cells change their firing patterns at both original and new sites parallel several studies that have focused on the spatial firing patterns of hippocampal place cells as animals explore multiple environments with overlapping features. These studies have shown that alterations in salient spatial or nonspatial cues often result in a "partial remapping" of the spatial firing patterns of hippocampal neurons, reflected in a combination of subsets of place cells that maintain their spatial firing patterns and other cells that cease firing or develop a new spatial firing pattern. Partial remapping has been observed when spatial cues are eliminated or reorganized (Shapiro et al., 1997), when rats move between environments with identical local spatial cues (Skaggs and McNaughton, 1998) and in the same environment when task demands are changed (Markus et al., 1995). Partial remapping could both distinguish experiences that share overlapping features and bind related experiences via coding elements that are common across representations (Eichenbaum, 1999). The learning-induced partial remapping at the overtrained goal locations may be essential in maintaining the structure of original and new location memories, and blocking this plasticity could result in the reconsolidation deficits observed in many studies (Debiec et al., 2002; Morris et al., 2006).

Other studies that have also included common stimuli or behavioral events in different places have observed hippocampal neurons that fire in response to the common events. Wood et al. (1999) reported similar firing patterns of hippocampal neurons when rats sampled the same odors in different places while performing a delayed non-match-to-sample task. Similarly, Singer et al. (2010) reported "path equivalence" of spatial firing patterns of hippocampal neurons as rats traversed separate but parallel segments of routes through a maze. Notably, in these experiments, and in several others in which rats traversed a common path segment while pursuing different trajectories (Frank et al., 2000; Wood et al., 2000; Ferbinteanu and Shapiro, 2003; Smith and Mizumori 2006; Bahar and Shapiro, 2012), some neurons have similar activity associated with the common events, whereas others fire distinctly, thus disambiguating overlapping memories. In monkeys (Hampson et al., 2004) and humans (Quiroga et al.,
2005), neurons have been observed that fire similarly in response to stimuli that are visually quite various but are similar in meaning (e.g., a cell that fires to various views of the same famous person and even the name of that person). Such "categorical" (Hampson et al., 2004; Lin et al., 2005) and "invariant" (Quiroga et al., 2005) firing patterns are consistent with the findings on rodents indicating that hippocampal neurons can link between related memories via common coding elements (Eichenbaum, 2004).

In the well-learned schema, the common coding of reward sites was not at the expense of pattern separation, although this was not the case for newly learned goal locations. The cells that fired in the new goal learning were those that fired at other goal sites, suggesting the incorporation of new cells into related networks. The stronger correlation between the new and original goal locations may share a common mechanism with the overrepresentation of the escape platform in a water-maze paradigm (Hollup et al., 2001; Fyhn et al., 2002). In those studies, when the goal was moved, some cells that fired at the original goal locations shifted firing to the new goal location, although only during the early phase of learning. Furthermore, just as we have reported, several of the cells had multiple fields on the annular water maze. However, because the goal location was moved - and not added - in the water-maze experiments, it is unclear whether cells in those studies came to relate the new and old goal locations through common firing.

We observed that, although cells fire at multiple locations during learning, the disambiguation of those locations takes days, potentially involving processes of consolidation. These results are reminiscent of previous studies that have shown that multiple exposures can be necessary for CA1 cells to distinguish arenas that differ only by the color of a cue card (Bostock et al., 1991) or remap in response to rotations of proximal and distal cues (Shapiro et al., 1997; Brown and Skaggs, 2002). When a rat must choose between one of several well-learned trajectories through the same space, the different trajectories are encoded by anticorrelated representations in CA1 (Bahar et al., 2011), suggesting that experience may increase the degree to which one representation suppresses competitors.

Common firing across locations was especially prominent during running through goals compared with waiting in the same locations. In mice (Kentros et al., 2004) and rats (Fenton et al., 2010), goal-directed behavior increases the spatial information (spatial discrimination) of hippocampal activity, and it has been suggested that decreases in spatial information during foraging may be attributable to multiple representations that are transiently expressed (Harris et al., 2003; Jackson and Redish, 2007; Kelemen and Fenton 2010; Jezek et al., 2011). Hok et al. (2007a) observed that a large proportion of the cells are active when rats run to an unmarked goal zone in an open arena and proposed that the activity reflected a transient reward prediction signal (Hok et al., 2007a,b). We also observed cell activity on arrival to goal sites, although this activity clearly signaled more than the possibility of reward because decoding of this activity led to the best estimate of a rat's location. It is unknown whether cell assemblies are composed of cells that fire in response to multiple related events or whether the separate goals are encoded by cells that can be part of distinct cell assemblies that are not simultaneously active. In either scenario, cell activity at multiple reward sites during running, and potentially on arrival to the goals, may reflect a decision-making process that requires transiently relating spatially separate events.

We hypothesize that learning in a familiar environment adds new hippocampal neurons to a preexisting neural network to 
support the memory of the relationship to the new memory with the old. The addition of new information to an existing schema causes reorganization of related networks; in our case, those that represent events at other goal locations. Learning also alters preexisting memory networks by incorporating new neurons into the ensemble that is active during recall. The reorganization of original memories to accommodate new information establishes new meaningful relationships and also maintains the preexisting similarities and differences of the original network. We propose that consolidation of new memories within preexisting networks is the reconsolidation of those existing networks, a process that ultimately stores new and original relationships within a common schema (McClelland et al., 1995; Tse et al., 2007; McKenzie and Eichenbaum, 2011).

\section{References}

Bahar AS, Shapiro ML (2012) Remembering to learn: independent place and journey coding mechanisms contribute to memory transfer. J Neurosci 32:2191-2203. CrossRef Medline

Bahar AS, Shirvalkar PR, Shapiro ML (2011) Memory-guided learning: CA1 and CA3 neuronal ensembles differentially encode the commonalities and differences between situations. J Neurosci 31:12270-12281. CrossRef Medline

Bartlett FC (1932) Remembering. Cambridge, UK: Cambridge UP.

Bostock E, Muller RU, Kubie JL (1991) Experience-dependent modifications of hippocampal place cell firing. Hippocampus 1:193-205. CrossRef Medline

Brown JE, Skaggs WE (2002) Concordant and discordant coding of spatial location in populations of hippocampal CA1 pyramidal cells. J Neurophysiol 88:1605-1613. Medline

Bunsey M, Eichenbaum H (1996) Conservation of hippocampal memory function in rats and humans. Nature 379:255-257. CrossRef Medline

Buzsáki G, Leung LW, Vanderwolf CH (1983) Cellular bases of hippocampal EEG in the behaving rat. Brain Res 287:139-171. Medline

Debiec J, LeDoux JE, Nader K (2002) Cellular and systems reconsolidation in the hippocampus. Neuron 36:527-538. CrossRef Medline

Dudai Y (2012) The restless engram: consolidations never end. Annu Rev Neurosci 35:227-247. CrossRef Medline

Dupret D, O’Neill J, Pleydell-Bouverie B, Csicsvari J (2010) The reorganization and reactivation of hippocampal maps predict spatial memory performance. Nat Neurosci 13:995-1002. CrossRef Medline

Dusek JA, Eichenbaum H (1997) The hippocampus and memory for orderly stimulus relations. Proc Natl Acad Sci U S A 94:7109-7114. CrossRef Medline

Eichenbaum H (1999) The hippocampus and mechanisms of declarative memory. Behav Brain Res 103:123-133. CrossRef Medline

Eichenbaum H (2004) Hippocampus: cognitive processes and neural representations that underlie declarative memory. Neuron 44:109-120. CrossRef Medline

Fenton AA, Lytton WW, Barry JM, Lenck-Santini PP, Zinyuk LE, Kubík S, Bures J, Poucet B, Muller RU, Olypher AV (2010) Attention-like modulation of hippocampus place cell discharge. J Neurosci 30:4613-4625. Medline

Ferbinteanu J, Shapiro ML (2003) Prospective and retrospective memory coding in the hippocampus. Neuron 40:1227-1239. CrossRef Medline

Frank LM, Brown EN, Wilson M (2000) Trajectory encoding in the hippocampus and entorhinal cortex. Neuron 27:169-178. CrossRef Medline

Fyhn M, Molden S, Hollup S, Moser MB, Moser E (2002) Hippocampal neurons responding to first-time dislocation of a target object. Neuron 35:555-566. CrossRef Medline

Geisler C, Robbe D, Zugaro M, Sirota A, Buzsáki G (2007) Hippocampal place cell assemblies are speed-controlled oscillators. Proc Natl Acad Sci U S A 104:8149-8154. CrossRef Medline

Gothard KM, Skaggs WE, Moore KM, McNaughton BL (1996) Binding of hippocampal CA1 neural activity to multiple reference frames in a landmark-based navigation task. J Neurosci 16:823-835. Medline

Hampson RE, Pons TP, Stanford TR, Deadwyler SA (2004) Categorization in the monkey hippocampus: a possible mechanism for encoding information into memory. Proc Natl Acad Sci U S A 101:3184-3189. CrossRef Medline
Hardt O, Einarsson EO, Nader K (2010) A bridge over troubled water: reconsolidation as a link between cognitive and neuroscientific memory research traditions. Annu Rev Psychol 61:141-167. CrossRef Medline

Harris KD, Csicsvari J, Hirase H, Dragoi G, Buzsáki G (2003) Organization of cell assemblies in the hippocampus. Nature 424:552-556. CrossRef Medline

Heckers S, Zalesak M, Weiss AP, Ditman T, Titone D (2004) Hippocampal activation during transitive inference in humans. Hippocampus 14:153-162. CrossRef Medline

Hok V, Lenck-Santini PP, Roux S, Save E, Muller RU, Poucet B (2007a) Goal-related activity in hippocampal place cells. J Neurosci 27:472-482. CrossRef Medline

Hok V, Lenck-Santini PP, Save E, Gaussier P, Banquet JP, Poucet B (2007b) A test of the time estimation hypothesis of place cell goal-related activity. J Integr Neurosci 6:367-378. Medline

Hollup SA, Molden S, Donnett JG, Moser MB, Moser EI (2001) Accumulation of hippocampal place fields at the goal location in an annular watermaze task. J Neurosci 21:1635-1644. Medline

Jackson J, Redish AD (2007) Network dynamics of hippocampal cellassemblies resemble multiple spatial maps within single tasks. Hippocampus 17:1209-1229. CrossRef Medline

Jezek K, Henriksen EJ, Treves A, Moser EI, Moser MB (2011) Theta-paced flickering between place-cell maps in the hippocampus. Nature 478:246-249. CrossRef Medline

Kelemen E, Fenton AA (2010) Dynamic grouping of hippocampal neural activity during cognitive control of two spatial frames. PLoS Biol 8:e1000403. CrossRef Medline

Kentros CG, Agnihotri NT, Streater S, Hawkins RD, Kandel ER (2004) Increased attention to spatial context increases both place field stability and spatial memory. Neuron 42:283-295. CrossRef Medline

Kobayashi T, Nishijo H, Fukuda M, Bures J, Ono T (1997) Task-dependent representations in rat hippocampal place neurons. J Neurophysiol 78: 597-613. Medline

Komorowski RW, Manns JR, Eichenbaum H (2009) Robust conjunctive item-place coding by hippocampal neurons parallels learning what happens where. J Neurosci 29:9918-9929. CrossRef Medline

Leutgeb S, Leutgeb JK, Barnes CA, Moser EI, McNaughton BL, Moser MB (2005) Independent codes for spatial and episodic memory in hippocampal neuronal ensembles. Science 309:619-623. CrossRef Medline

Lin L, Osan R, Shoham S, Jin W, Zuo W, Tsien JZ (2005) Identification of network-level coding units for real-time representation of episodic experiences in the hippocampus. Proc Natl Acad Sci U S A 102:6125-6130. CrossRef Medline

MacDonald CJ, Lepage KQ, Eden UT, Eichenbaum H (2011) Hippocampal "time cells" bridge the gap in memory for discontiguous events. Neuron 71:737-749. CrossRef Medline

Markus EJ, Qin YL, Leonard B, Skaggs WE, McNaughton BL, Barnes CA (1995) Interactions between location and task affect the spatial and directional firing of hippocampal neurons. J Neurosci 15:7079-7094. Medline

McClelland JL, McNaughton BL, O'Reilly RC (1995) Why there are complementary learning systems in the hippocampus and neocortex: insights from the successes and failures of connectionist models of learning and memory. Psychol Rev 102:419-457. CrossRef Medline

McKenzie S, Eichenbaum H (2011) Consolidation and reconsolidation: two lives of memories? Neuron 71:224-233. CrossRef Medline

McNaughton BL, Barnes CA, O'Keefe J (1983) The contributions of position, direction, and velocity to single unit activity in the hippocampus of freely-moving rats. Exp Brain Res 52:41-49. Medline

Morris RG, Inglis J, Ainge JA, Olverman HJ, Tulloch J, Dudai Y, Kelly PA (2006) Memory reconsolidation: sensitivity of spatial memory to inhibition of protein synthesis in dorsal hippocampus during encoding and retrieval. Neuron 50:479-489. CrossRef Medline

O’Neill J, Senior T, Csicsvari J (2006) Place-selective firing of CA1 pyramidal cells during sharp wave/ripple network patterns in exploratory behavior. Neuron 49:143-155. CrossRef Medline

Piaget J (1926) The language and thought of the child. New York: Harcourt, Brace.

Piaget J (1929) The child's conception of the world (Tomlinson J, Thomlinson A, translators). New York: Harcourt, Brace.

Quiroga RQ, Reddy L, Kreiman G, Koch C, Fried I (2005) Invariant visual 
representation by single neurons in the human brain. Nature 435:11021107. CrossRef Medline

Shapiro ML, Tanila H, Eichenbaum H (1997) Cues that hippocampal place cells encode: dynamic and hierarchical representation of local and distal stimuli. Hippocampus 7:624-642. CrossRef Medline

Singer AC, Karlsson MP, Nathe AR, Carr MF, Frank LM (2010) Experiencedependent development of coordinated hippocampal spatial activity representing the similarity of related locations. J Neurosci 30:11586-11604. CrossRef Medline

Skaggs WE, McNaughton BL (1998) Spatial firing properties of hippocampal CA1 populations in an environment containing two visually identical regions. J Neurosci 18:8455-8466. Medline

Smith DM, Mizumori SJ (2006) Learning-related development of contextspecific neuronal responses to places and events: the hippocampal role in context processing. J Neurosci 26:3154-3163. CrossRef Medline

Speakman A, O'Keefe J (1990) Hippocampal complex spike cells do not change their place fields if the goal is moved within a cue controlled environment. Eur J Neurosci 2:544-555. Medline

Trullier O, Shibata R, Mulder AB, Wiener SI (1999) Hippocampal neuronal position selectivity remains fixed to room cues only in rats alternating between place navigation and beacon approach tasks. Eur J Neurosci 11:4381-4388. Medline

Tse D, Langston RF, Kakeyama M, Bethus I, Spooner PA, Wood ER, Witter MP, Morris RG (2007) Schemas and memory consolidation. Science 316:76-82. CrossRef Medline

Tse D, Takeuchi T, Kakeyama M, Kajii Y, Okuno H, Tohyama C, Bito H,
Morris RGM (2011) Schema-dependent gene activation and memory encoding in neocortex. Science 333:891-895. CrossRef Medline

van Kesteren MT, Ruiter DJ, Fernández G, Henson RN (2012) How schema and novelty augment memory formation. Trends Neurosci 35:211-219. Medline

Wirth S, Yanike M, Frank LM, Smith AC, Brown EN, Suzuki WA (2003) Single neurons in the monkey hippocampus and learning of new associations. Science 300:1578-1581. CrossRef Medline

Wood ER, Dudchenko PA, Eichenbaum H (1999) The global record of memory in hippocampal neuronal activity. Nature 397:613-616. CrossRef Medline

Wood ER, Dudchenko PA, Robitsek RJ, Eichenbaum H (2000) Hippocampal neurons encode information about different types of memory episodes occurring in the same location. Neuron 27:623-633. CrossRef Medline

Zeithamova D, Preston AR (2010) Flexible memories: differential roles for medial temporal lobe and prefrontal cortex in cross-episode binding. J Neurosci 30:14676-14684. CrossRef Medline

Zeithamova D, Dominick AL, Preston AR (2012) Hippocampal and ventral medial prefrontal activation during retrieval-mediated learning supports novel inference. Neuron 75:168-179. CrossRef Medline

Zhang K, Ginzburg I, McNaughton BL, Sejnowski TJ (1998) Interpreting neuronal population activity by reconstruction: unified framework with application to hippocampal place cells. J Neurophysiol 79:1017-1044. Medline 